\title{
Photoacoustic image reconstruction in Bayesian framework
}

Jenni Tick, Aki Pulkkinen, Felix Lucka, Robert Ellwood, Ben T. Cox, et al.

Jenni Tick, Aki Pulkkinen, Felix Lucka, Robert Ellwood, Ben T. Cox, Simon R. Arridge, Tanja Tarvainen, "Photoacoustic image reconstruction in Bayesian framework," Proc. SPIE 10494, Photons Plus Ultrasound: Imaging and Sensing 2018, 1049450 (19 February 2018); doi: 10.1117/12.2288163

SPIE. Event: SPIE BiOS, 2018, San Francisco, California, United States 


\title{
Photoacoustic image reconstruction in Bayesian framework
}

\author{
Jenni Tick ${ }^{\mathrm{a}}$, Aki Pulkkinen ${ }^{\mathrm{a}}$, Felix Lucka ${ }^{\mathrm{b}, \mathrm{c}}$, Robert Ellwood ${ }^{\mathrm{d}}$, Ben T. Cox ${ }^{\mathrm{d}}$, Simon R. \\ Arridge $^{\mathrm{c}}$, and Tanja Tarvainen ${ }^{\mathrm{a}, \mathrm{c}}$ \\ ${ }^{a}$ Department of Applied Physics, University of Eastern Finland, P.O. Box 1627, 70211 Kuopio, \\ Finland \\ ${ }^{\mathrm{b}}$ Centrum Wiskunde \& Informatica, P.O. Box 94079, 1090 GB Amsterdam, Netherlands \\ ${ }^{\mathrm{c}}$ Department of Computer Science, University College London, Gower Street, London WC1E \\ 6BT, United Kingdom \\ ${ }^{\mathrm{d} D e p a r t m e n t ~ o f ~ M e d i c a l ~ P h y s i c s ~ a n d ~ B i o m e d i c a l ~ E n g i n e e r i n g, ~ U n i v e r s i t y ~ C o l l e g e ~ L o n d o n, ~}$ \\ Gower Street, London WC1E 6BT, United Kingdom
}

\begin{abstract}
The photoacoustic image reconstruction problem (inverse problem) is to estimate an initial acoustic pressure distribution from measurements of ultrasound waves generated within an object due to optical excitation with a short light pulse. In this work, the recently suggested Bayesian approach to photoacoustic tomography is extended to three dimensions and an iterative matrix-free method for the solution of the problem is described. Image reconstruction is investigated with numerical simulations and experimental data. The use of different prior information and noise models in different sensor geometries, including a limited-view setup, is investigated. The results show that the Bayesian approach can produce accurate estimates of the initial pressure distribution even in a limited-view setup provided that prior information and the noise have been properly modelled.
\end{abstract}

Keywords: photoacoustic tomography, inverse problems, Bayesian methods

\section{INTRODUCTION}

Photoacoustic tomography (PAT) $)^{1-7}$ is a hybrid imaging modality in which the imaging contrast is based on optical properties whereas the imaging resolution is based on acoustic detection. In PAT measurement, the region of tissue of interest is irradiated with a short visible or near-infrared light pulse, which results in the generation of pressure waves via photoacoustic effect. The propagating pressure waves are measured by ultrasound sensors at several positions outside the imaged tissue. In the inverse problem of PAT, the initial pressure distribution, which is related to absorbed optical energy density, is reconstructed from the measured photoacoustic time series.

Various approaches for photoacoustic image reconstruction have been developed. ${ }^{8-24}$ Recently, utilizing a Bayesian approach in PAT was proposed. ${ }^{24}$ In this work, the Bayesian approach for photoacoustic image reconstruction is extended to three spatial dimensions (3D). Due to the large dimension of the problem, the matrix presentation that was used in two dimensions can no longer be applied. Therefore, maximum a posteriori (MAP) estimates for image reconstruction are computed iteratively in a matrix-free form utilizing the acoustic forward operator and its adjoint. Image reconstruction is investigated using different distributions of Gaussian prior and noise models in various imaging situations including limited-view measurement setup. In addition to numerical simulations, the feasibility of the technique is demonstrated with experimental data.

Further author information: (Send correspondence to J.T.)

J.T.: E-mail: jenni.tick@uef.fi, Telephone: +358 505643669

Photons Plus Ultrasound: Imaging and Sensing 2018, edited by Alexander A. Oraevsky, Lihong V. Wang, Proc. of SPIE Vol. 10494, 1049450 · @ 2018 SPIE · CCC code: 1605-7422/18/\$18 · doi: 10.1117/12.2288163 


\section{PHOTOACOUSTIC MODEL}

In PAT, absorbed energy originating from a short light pulse causes thermoelastic expansion of the tissue which in turn leads to a pressure wave propagating through the object. In a homogeneous medium, this pressure wave can be described by a wave equation

$$
\begin{aligned}
\left(\frac{\partial^{2}}{\partial t^{2}}-c^{2} \nabla^{2}\right) p(r, t) & =0 \\
p(r, t=0) & =p_{0}(r) \\
\frac{\partial}{\partial t} p(r, t=0) & =0,
\end{aligned}
$$

where $p$ is the pressure, $r$ is the spatial position, $t$ is the time, $c$ is the speed of sound and $p_{0}$ is the initial pressure distribution. ${ }^{1,2,4}$ The pressure waves propagate to the surface of the imaged object where they are measured as a function of time by ultrasound sensors.

In practice, the measured pressure time series are polluted with noise, which is assumed to be additive in this work. The discrete observation model with an additive noise model for PAT is

$$
p_{t}=K p_{0}+e,
$$

where $p_{t}$ is the measured acoustic pressure time series, $p_{0}$ is the discrete initial pressure distribution, $K$ is the discrete acoustic forward operator that maps the initial pressure distribution to measurable data by Eq. (1), and $e$ denotes the measurement noise.

\section{BAYESIAN APPROACH FOR IMAGE RECONSTRUCTION}

In this work, reconstruction of the initial pressure $p_{0}$ is based on the Bayesian framework ${ }^{25,26}$ with Gaussian assumptions. The Bayesian approach is a statistical estimation method in which measurements, model and prior information are used to infer the probability distribution of the parameter of primary interest. From this distribution, point estimates for image reconstruction can be computed. In this work, the MAP estimate is considered as an estimate for the initial pressure. The MAP estimate can be formally computed as ${ }^{24}$

$$
p_{0, \mathrm{MAP}}=\left(K^{\mathrm{T}} \Gamma_{e}^{-1} K+\Gamma_{p_{0}}^{-1}\right)^{-1}\left(K^{\mathrm{T}} \Gamma_{e}^{-1}\left(p_{t}-\eta_{e}\right)+\Gamma_{p_{0}}^{-1} \eta_{p_{0}}\right),
$$

where $\eta_{e}$ and $\Gamma_{e}$ are the mean vector and covariance matrix characterizing the noise, and $\eta_{p_{0}}$ and $\Gamma_{p_{0}}$ are the mean vector and covariance matrix of the prior information.

Due to the large dimensions of the problem in 3D, the matrices in Eq. (3) cannot be explicitly constructed. Therefore, the MAP estimate is solved iteratively using linear solvers of MATLAB in a matrix-free form. Equation (3) is expressed in equivalent form as a linear system

$$
\left(\Gamma_{p_{0}} K^{\mathrm{T}} \Gamma_{e}^{-1} K+I\right) p_{0, \mathrm{MAP}}=\Gamma_{p_{0}} K^{\mathrm{T}} \Gamma_{e}^{-1}\left(p_{t}-\eta_{e}\right)+\eta_{p_{0}},
$$

where $I$ is an identity matrix. Equation (4) is obtained from (3) by multiplying it with $K^{\mathrm{T}} \Gamma_{e}^{-1} K+\Gamma_{p_{0}}^{-1}$ and $\Gamma_{p_{0}}$. The form in (4) avoids inversion of a large system matrix $K^{\mathrm{T}} \Gamma_{e}^{-1} K+\Gamma_{p_{0}}$ and the prior covariance matrix $\Gamma_{p_{0}}$. In the iteration, the forward model operator $K$ is replaced by solving wave equation (1) using the k-space time domain method implemented with the k-Wave MATLAB toolbox. ${ }^{27}$ The transpose of the forward model, $K^{T}$, is computed using an adjoint wave equation as described in Ref. 28. The adjoint is solved using k-Wave.

\subsection{Prior Information}

In this work, two different Gaussian priors: the white noise prior and Ornstein-Uhlenbeck process ${ }^{29}$ were studied. In the white noise prior, the covariance matrix is a diagonal matrix with the values of variance $\sigma_{p_{0}}^{2}$ on the diagonal

$$
\Gamma_{p_{0}}=\sigma_{p_{0}}^{2} I .
$$




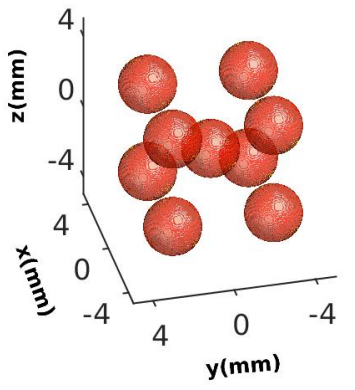

(a)

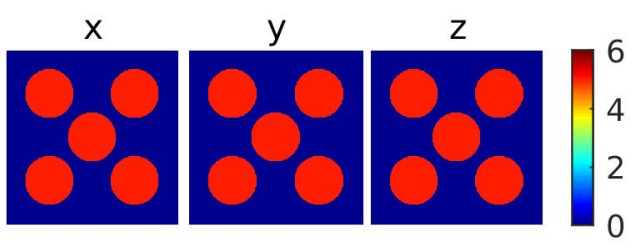

(b)

Figure 1. The simulated (true) initial pressure distribution. (a) The locations of the simulated spheres. (b) The maximum intensity projections along the axis directions $x, y$ and $z$.

The white noise prior is well suited for the estimation of parameters which have a non-smooth spatial distribution, since it assumes that the estimated parameters are independent of each other or have no spatial correlation. However, in typical applications of PAT some spatial correlation in parameter values between the pixels can be expected. Therefore, the Ornstein-Uhlenbeck prior, which promotes distributions that can be locally close to homogeneous with sharp changes between different areas, could be a more realistic choice of prior. The covariance matrix of the Ornstein-Uhlenbeck prior is defined as

$$
\Gamma_{p_{0}, i j}=\sigma_{p_{0}}^{2} \exp \left(-\frac{\left\|r_{i}-r_{j}\right\|}{l}\right)
$$

where $i$ and $j$ are the pixel indices, $r_{i}$ and $r_{j}$ are the corresponding pixel positions, $\sigma_{p_{0}}$ is the variance and $l$ is the characteristic length scale which controls the spatial range of correlation.

\section{NUMERICAL SIMULATIONS}

The Bayesian approach to 3D photoacoustic image reconstruction was tested with simulations. The impact of both prior information and noise model on the reconstructed initial pressure distributions were investigated.

The simulated domain was a three dimensional cube with side length of $10 \mathrm{~mm}$. The domain was assumed to be non-attenuating and homogenous with a constant speed of sound $\mathrm{c}=1500 \mathrm{~m} / \mathrm{s}$. The true simulated initial pressure distribution consisted of nine spheres of $1.43 \mathrm{~mm}$ radius on a homogenous background. Eight of these spheres were located near the corners of the cube and one sphere was located in the middle of the cube. The initial acoustic pressure was set to be 5 on the spheres and zero elsewhere. Figure 1 shows the simulated initial pressure distribution. Data was simulated in a full-view and limited-view sensor geometry. In the full-view setup, 62119 acoustic sensors were located across all sides of the cube and in the limited-view setup 10404 sensors were located only on one side (side in the direction of the positive $\mathrm{z}$ axis) of the domain. The acoustic pressure at the sensors as function of time was calculated using the k-Wave MATLAB toolbox. ${ }^{27}$ The pressure signals were recorded for $14.1 \mu \mathrm{s}$ at $60 \mathrm{MHz}$ (i.e. 849 time samples were simulated), which corresponds to an acoustic propagation distance of $21.15 \mathrm{~mm}$. Noisy measurement were formed by adding uncorrelated Gaussian distributed noise with zero mean and a standard deviation set to $1 \%$ of the peak amplitude of the simulated pressure signal to recorded pressure signals. In the data simulation, $306^{3}$ cubic voxels with the side length $\Delta h=32.7 \mu \mathrm{m}$ were used.

For the image reconstruction, the MAP estimates were computed iteratively using Eq. (4) as described in Sec. 3. In the image reconstruction, sparser discretization consisting of $204^{3}$ voxels $(\Delta h=49 \mu \mathrm{m})$ was used. The effect of accurate and inaccurate modelling of noise and prior statistics on the reconstruction was studied. The measurement noise was considered to be uncorrelated Gaussian distributed noise with zero mean and the standard deviation set to $0.1,0.5,1$ (the correct noise level), 5 and $10 \%$ of the peak positive amplitude of the 


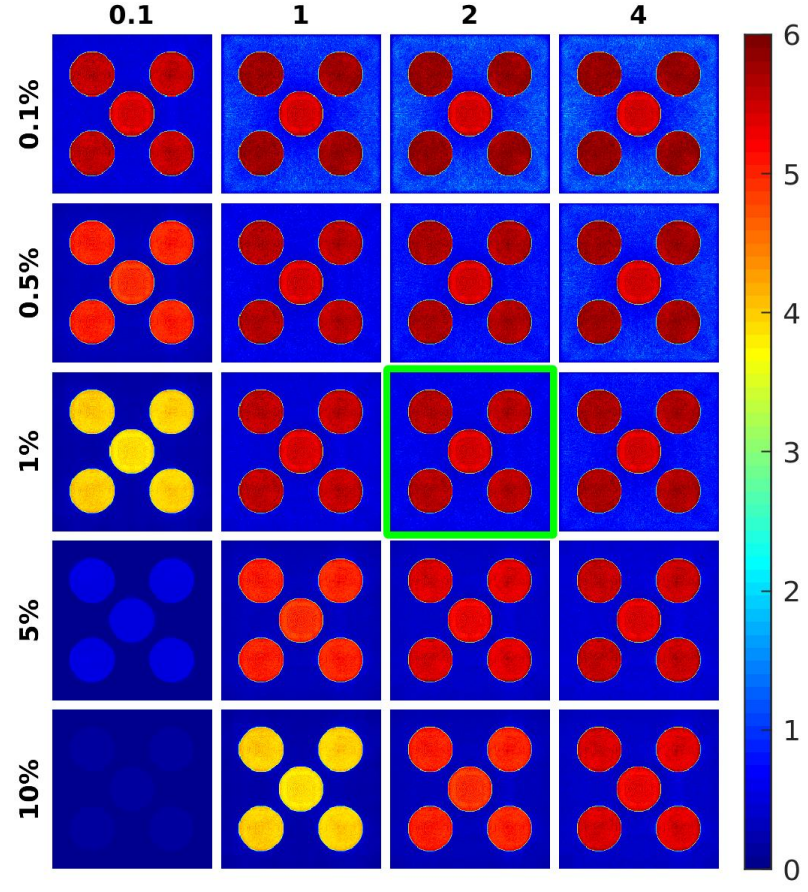

(a)

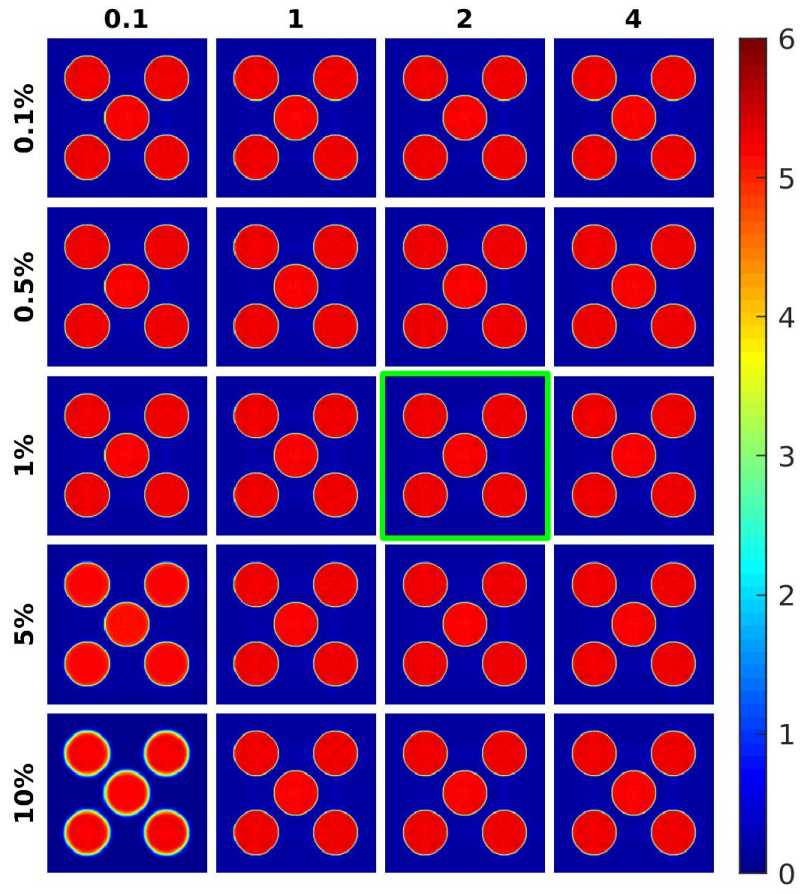

(b)

Figure 2. Maximum intensity projections of the MAP estimates along $x$ axis using different standard deviations of the noise and prior in the full-view setup. (a) The white noise prior is used. (b) The Ornstein-Uhlenbeck prior is used. In the images (a) and (b), the standard deviation of the prior is 0.1 (first column), 1 (second column), 2 (third column) and 4 (fourth column), and the standard deviation of the noise is 0.1 (first row), 0.5 (second row), 1 (third row), 5 (fourth row) or $10 \%$ (fifth row) of the peak positive amplitude of the noisy simulated data. The reconstruction obtained with the correct noise level of $1 \%$ and the most suitable prior standard deviation of 2 are indicated with green square.

noisy simulated data. As prior information, the white noise and Ornstein-Uhlenbeck priors were used. The means of the priors were set to the value of the background (zero) and the standard deviations were set to 0.1, 1, 2 or 4 . The prior standard deviation of 2 corresponds to the assumption that $99.7 \%$ of the initial pressure values are expected to be in the range between -6 and 6 . For the Ornstein-Uhlenbeck prior, the characteristic length scale $l=0.49 \mathrm{~mm}$ was used. The computations were performed on a GPU. The estimates were compared quantitatively by computing the relative errors between the simulated (true) and estimated initial pressure distributions

$$
E_{p_{0}}=100 \% \cdot \frac{\left\|p_{0, \mathrm{TRUE}}-p_{0, \mathrm{MAP}}\right\|}{\left\|p_{0, \mathrm{TRUE}}\right\|},
$$

where $p_{0, \text { TRUE }}$ is the simulated initial pressure distribution interpolated to the reconstruction space and $p_{0, \mathrm{MAP}}$ are the estimated values.

\subsection{Results}

The reconstructions obtained using different standard deviations of the noise and prior in the full-view sensor geometry are shown in Fig. 2 for the white noise and Ornstein-Uhlenbeck priors. It can be seen that reconstructions obtained using the full-view sensor geometry look qualitatively very similar to the true initial pressure distribution almost at all standard deviation combinations of the noise and prior. The locations, sizes and shapes of the spherical inclusions are reconstructed accurately. In the case of the Ornstein-Uhlenbeck prior, the choice of the standard deviation of the noise and prior hardly affects the quality or quantitative values of the reconstructions. Also, in the case of the white noise prior, only the large standard deviations of the noise and small 


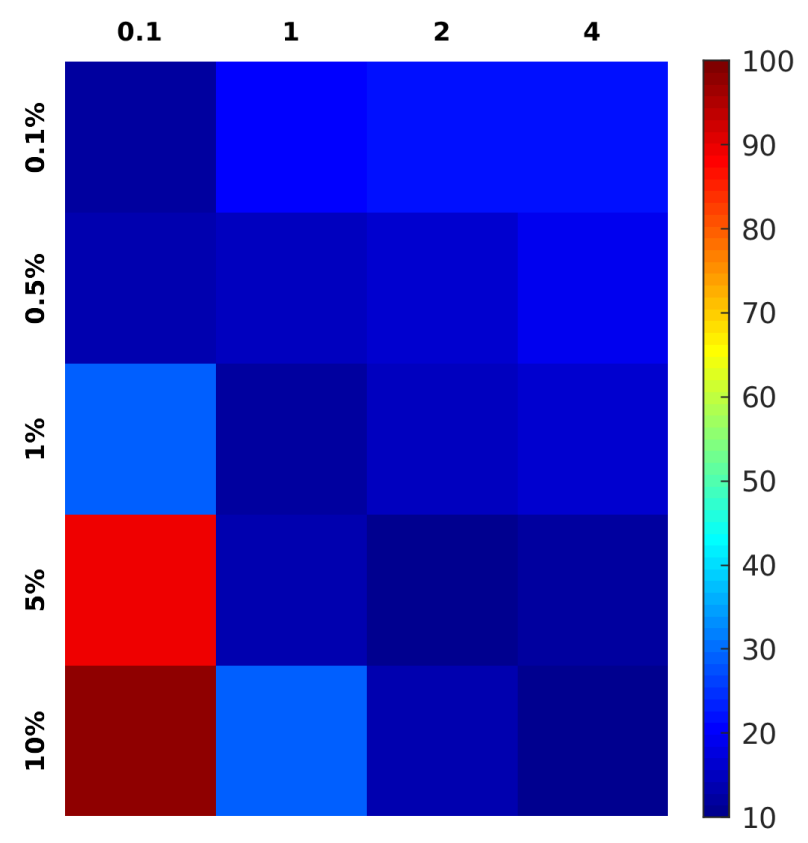

(a)

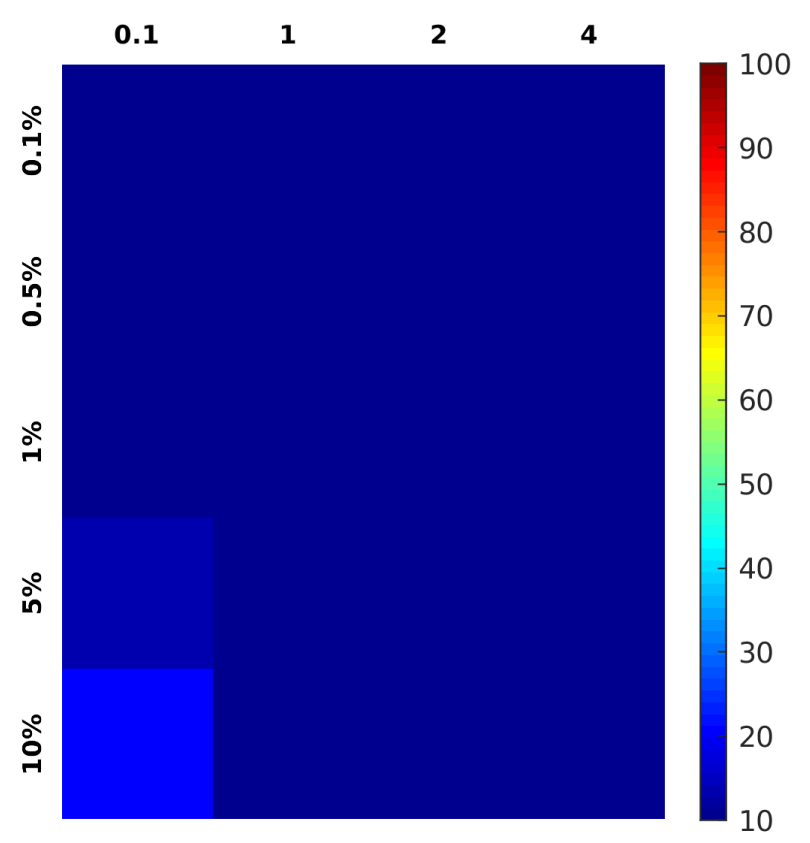

(b)

Figure 3. Visualization of the relative errors of the MAP estimates (in percentage) calculated in the full-view sensor geometry using different standard deviations of the noise and prior. (a) The white noise prior is used. (b) The OrnsteinUhlenbeck prior is used. In the images (a) and (b), the standard deviation of the prior is 0.1 (first column), 1 (second column), 2 (third column) and 4 (fourth column), and the standard deviation of the noise is 0.1 (first row), 0.5 (second row), 1 (third row), 5 (fourth row) or $10 \%$ (fifth row) of the peak positive amplitude of the noisy simulated data.

standard deviations of the prior have a noticeable effect on reconstructions. The relative errors of the estimates are visualized in the Fig. 3. The relative errors of the estimates obtained using the Ornstein-Uhlenbeck prior are slightly smaller than relative errors of the estimates obtained using the white noise prior. In addition, the relative errors obtained using the Ornstein-Uhlenbeck prior have smaller variation in their values than the relative errors obtained using the white noise prior.

The reconstructions obtained using different standard deviations of the noise and prior in the limited-view sensor geometry are shown in Fig. 4 for the white noise and Ornstein-Uhlenbeck priors. In the limited-view sensor geometry, the quality of the images is reduced compared to the full-view reconstructions. Artefacts and distortions can be seen and they are more severe at larger distances from the sensor surface (located on the right of the maximum intensity projections). The MAP estimates obtained using the Ornstein-Uhlenbeck prior look qualitatively better than the estimates obtained using the white noise prior. In the case of the OrnsteinUhlenbeck prior, the visual appearance and the quantitative values of the estimates stay approximately the same even if the different standard deviations of the noise and prior are considered. When the white noise prior is used, the values of the standard deviations of the noise and prior severely affect the qualitative and quantitative values of the reconstructions. The relative errors of the estimates are visualized in the Fig. 5. As can be seen, the relative errors of the estimates obtained using the limited-view sensor geometry have larger values than the relative errors of the estimates obtained using the full-view sensor geometry. In addition, the relative errors of the estimates obtained using the white noise prior are significantly larger than the relative errors of the estimates obtained using the Ornstein-Uhlenbeck prior. Also, the range of the values of the relative errors obtained using the white prior is larger than the range of the values of the relative errors obtained using the Ornstein-Uhlenbeck prior. 


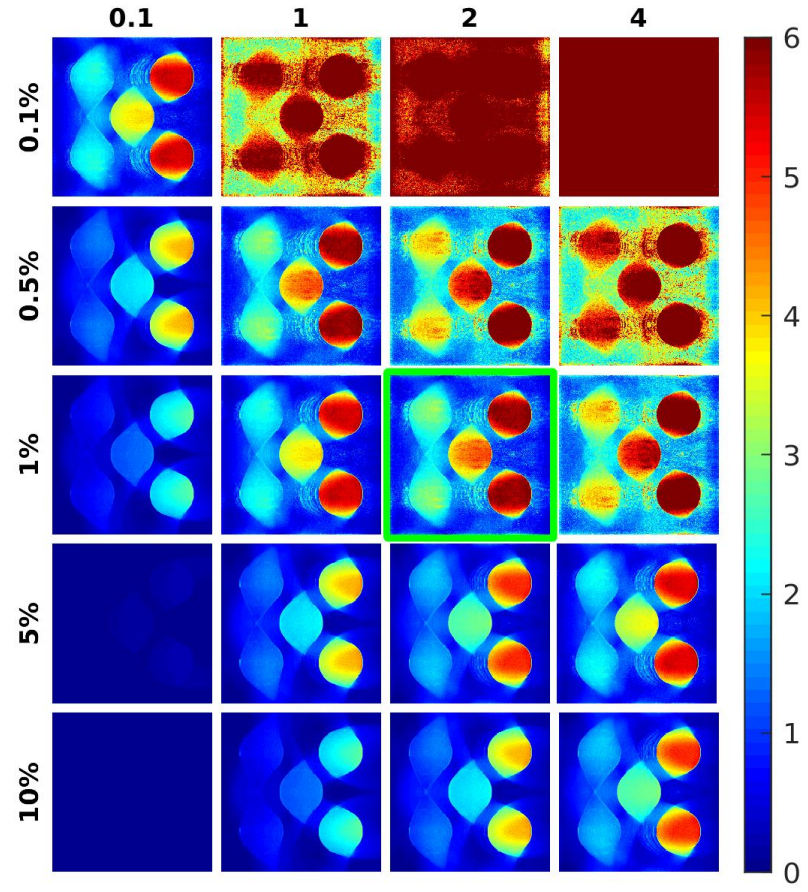

(a)

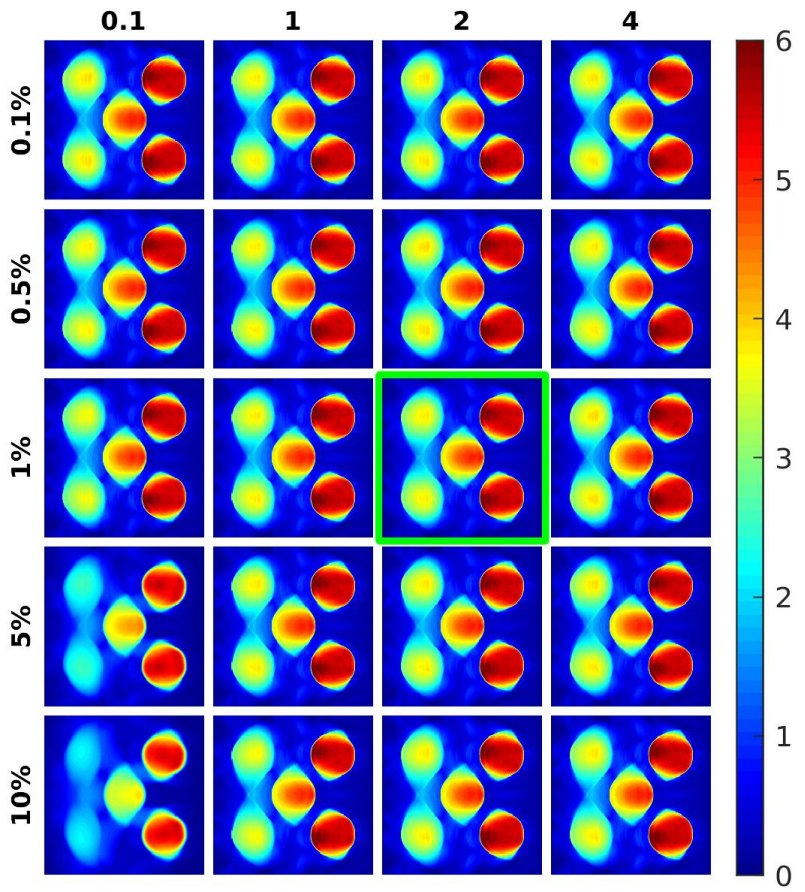

(b)

Figure 4. Maximum intensity projections of the MAP estimates along $x$ axis using different standard deviations of the noise and prior in the limited-view setup. (a) The white noise prior is used. (b) The Ornstein-Uhlenbeck prior is used. In the images (a) and (b), the standard deviation of the prior is 0.1 (first column), 1 (second column), 2 (third column) and 4 (fourth column), and the standard deviation of the noise is 0.1 (first row), 0.5 (second row), 1 (third row), 5 (fourth row) or $10 \%$ (fifth row) of the peak positive amplitude of the noisy simulated data. The reconstruction obtained with the correct noise level of $1 \%$ and the most suitable prior standard deviation of 2 are indicated with green square.

\section{EXPERIMENTAL STUDIES}

In addition to numerical simulations, the approach was tested with experimental data obtained from a strand phantom. The phantom consisted of three black polymer strands mounted to a custom polymethyl methacrylate (PMMA) mount immersed in water. A photograph of the phantom is shown in Fig. 6. The phantom was imaged with a photoacoustic measurement system ${ }^{30,31}$ developed in the Photoacoustic Imaging Group of University College London. The excitation light source was a Nd:YAG laser operating at $1064 \mathrm{~nm}$ with a pulse duration of 8 ns. The generated photoacoustic signals were measured using a single planar Fabry-Pérot sensor (a nominal 3 $\mathrm{dB}$ bandwidth of $39 \mathrm{MHz}$ ). In the measurement, approximately an area of $10 \mathrm{~mm} \times 10 \mathrm{~mm}$ on the sensor was scanned with a step size of $100 \mu \mathrm{m}$. More details on the phantom, experimental setup and measurements can be found in Ref. 31.

Image reconstruction was performed by computing the MAP estimate using the Eq. (4) as described in Sec. 3. Before reconstructions, the measured pressure signals were filtered using a bandpass filter with cutoff frequencies between 0.5 and $20 \mathrm{MHz}$ to remove nuisance signal components such as a rising trend of the measured pressure signals. Bandpass filtering was also taken into account in the modelling. ${ }^{24}$ The reconstruction domain was a rectangular volume that was discretized by $226 \times 204 \times 198$ voxels with side length of $\Delta h=50 \mu \mathrm{m}$. In the reconstructions, the sound speed of the medium was assumed to be $1485 \mathrm{~m} / \mathrm{s}$. The measurement noise was characterized in each sensor by determining the mean and standard deviation from a time frame of measured signal that contains only noise. As prior information, the white noise and Ornstein-Uhlenbeck priors were used. The mean $\eta_{p_{0}}=0$ and standard deviation $\sigma_{p_{0}}=0.25$ was utilized for both priors. For the Ornstein-Uhlenbeck prior, the characteristic length scale $l=0.25 \mathrm{~mm}$ was used. 


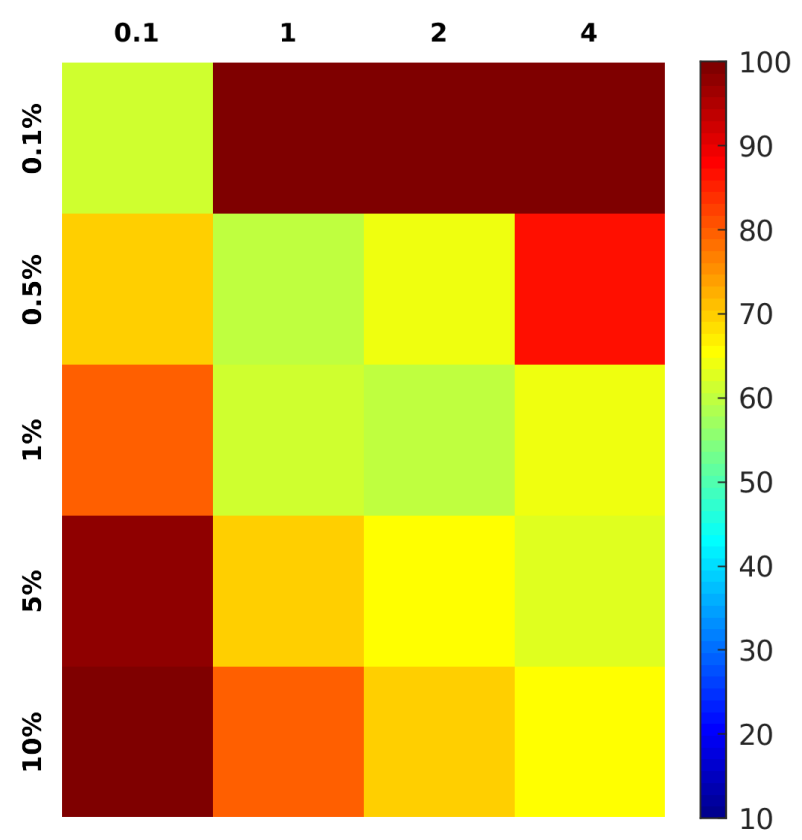

(a)

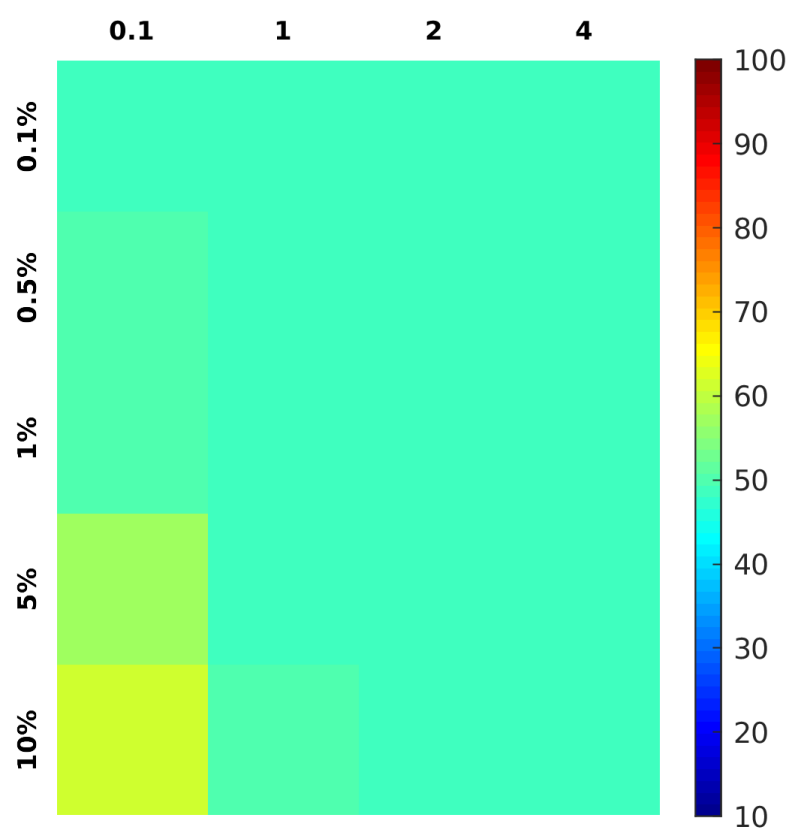

(b)

Figure 5. Visualization of the relative errors of the MAP estimates (in percentage) calculated in the limited-view sensor geometry using different standard deviations of the noise and prior. (a) The white noise prior is used. (b) The OrnsteinUhlenbeck prior is used. In the images (a) and (b), the standard deviation of the prior is 0.1 (first column), 1 (second column), 2 (third column) and 4 (fourth column), and the standard deviation of the noise is 0.1 (first row), 0.5 (second row), 1 (third row), 5 (fourth row) or $10 \%$ (fifth row) of the peak positive amplitude of the noisy simulated data.

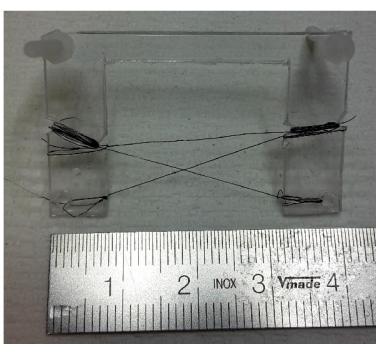

(a)

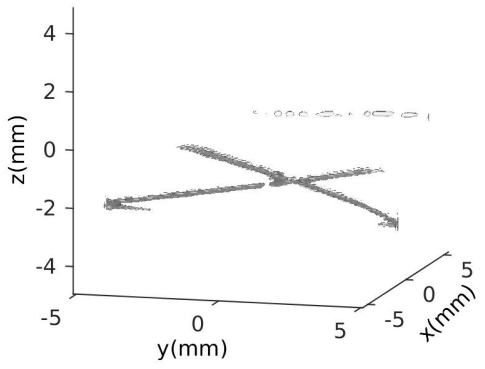

(b)

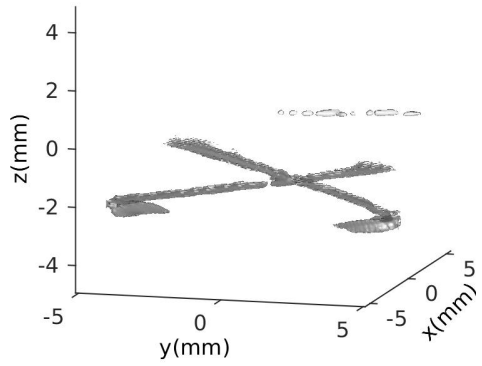

(c)

Figure 6. (a) A photograph of the strand phantom. (b) The contour surface of the reconstructed image obtained using the white noise prior. (c) The contour surface of the reconstructed image obtained using the Ornstein-Uhlenbeck prior.

\subsection{Results}

Contour surfaces of the reconstructed images obtained using the white noise and Ornstein-Uhlenbeck prior are presented in Fig. 6. Figure 7 shows the maximum intensity projections of the reconstructions. As it can be seen in these visualizations, the reconstructions look visually equally good. All three strands can be distinguished from both reconstructions. However, some artefacts caused by limited-view measurement setup or modelling errors are visible. 


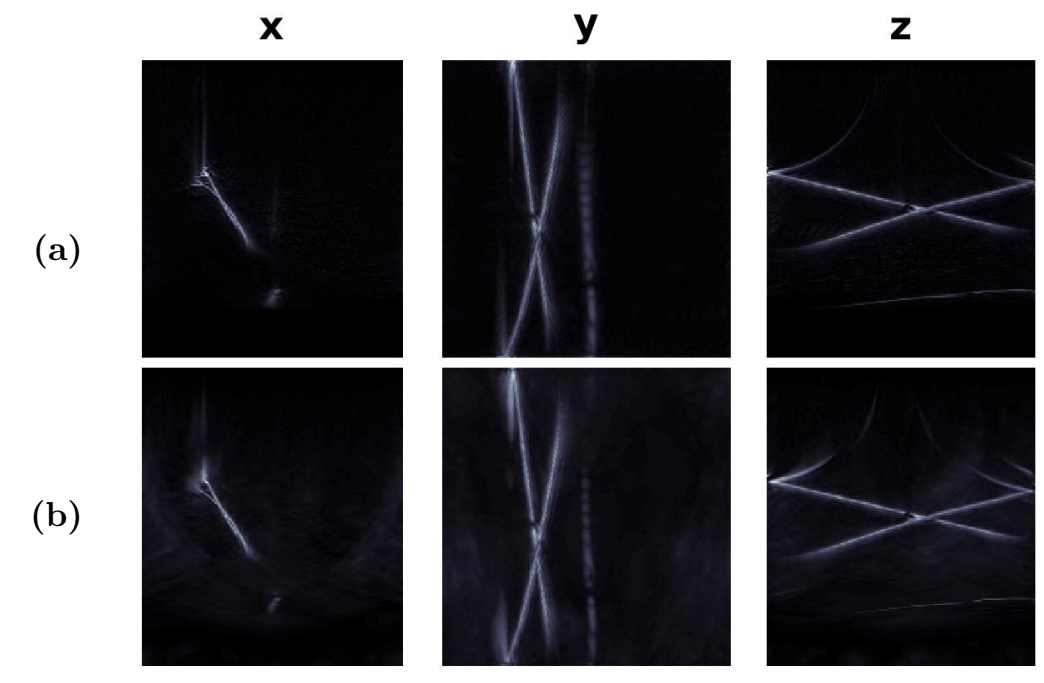

Figure 7. Maximum intensity projections of the photoacoustic images of the strand phantom along axis directions $x, y$ and $z$. (a) The white noise prior is used. (b) The Ornstein-Uhlenbeck prior is used.

\section{CONCLUSIONS}

An iterative matrix-free method for 3D photoacoustic image reconstruction based on the Bayesian approach was described. The approach was investigated with numerical simulations with different noise and prior statistics simulating the accurate and inaccurate information on the measurement noise and target. MAP estimates were computed for two different sensor geometries. The simulations show that both the quality of the reconstructed images and the quantitative accuracy of the estimates depend on the choice of the noise statistics and prior information. This is especially evident in the limited-view measurement setup. The approach was also tested with experimental data. The reconstructed images represented the features of the imaged object showing that the Bayesian framework can be applied to PAT image reconstruction with experimental data. In this work, only photoacoustic image reconstruction using the Bayesian approach was considered. However, in the Bayesian framework,uncertainty quantification is also possible. Extending the work to consider reliability of the estimates will be considered in the future.

\section{ACKNOWLEDGMENTS}

This work has been supported by the Academy of Finland (projects 286247, 314411 and 250215 Finnish Centre of Excellence in Inverse Problems Research), Instrumentarium Science Foundation, and Jane and Aatos Erkko foundation. FL acknowledges financial support from EPSRC project EP/K009745/1 "Dynamic High Resolution Photoacoustic Tomography System" and of the Netherlands Organization for Scientific Research (NWO), project nr. 613.009.106/2383. In addition, we gratefully acknowledge the support of NVIDIA Corporation with donation of Tesla K40 GPU used for this research.

\section{REFERENCES}

[1] Xu, M. and Wang, L. V., "Photoacoustic imaging in biomedicine," Review of Scientific Instruments 77(4), 041101 (2006).

[2] Li, C. and Wang, L. V., "Photoacoustic tomography and sensing in biomedicine," Physics in Medicine and Biology 54(19), R59-R97 (2009).

[3] Beard, P., "Biomedical photoacoustic imaging," Interface Focus 1(4), 602-631 (2011).

[4] Lutzweiler, C. and Razansky, D., "Optoacoustic imaging and tomography: Reconstruction approaches and outstanding challenges in image performance and quantification," Sensors (Switzerland) 13(6), 7345-7384 (2013). 
[5] Xia, J. and Wang, L. V., "Small-animal whole-body photoacoustic tomography: a review," IEEE Transactions on Biomedical Engineering 61(5), 1380-1389 (2014).

[6] Wang, L. V. and Yao, J., "A practical guide to photoacoustic tomography in the life sciences," Nature Methods 13(8), 627-638 (2016).

[7] Zhou, Y., Yao, J., and Wang, L. V., "Tutorial on photoacoustic tomography," Journal of Biomedical Optics 21(6), 061007-061007 (2016).

[8] Finch, D., Patch, S. K., and Rakesh, "Determining a function from its mean values over a family of spheres," SIAM Journal on Mathematical Analysis 35(5), 1213-1240 (2004).

[9] Xu, M. and Wang, L. V., "Universal back-projection algorithm for photoacoustic computed tomography," Physical Review E - Statistical, Nonlinear, and Soft Matter Physics 71(1), 016706 (2005).

[10] Agranovsky, M. and Kuchment, P., "Uniqueness of reconstruction and an inversion procedure for thermoacoustic and photoacoustic tomography with variable sound speed," Inverse Problems 23(5), 2089-2102 (2007).

[11] Kunyansky, L. A., "A series solution and a fast algorithm for the inversion of the spherical mean Radon transform," Inverse Problems 23(6), S11-S20 (2007).

[12] Burgholzer, P., Matt, G. J., Haltmeier, M., and Paltauf, G., "Exact and approximative imaging methods for photoacoustic tomography using an arbitrary detection surface," Physical Review E - Statistical, Nonlinear, and Soft Matter Physics 75(4), 046706 (2007).

[13] Hristova, Y., Kuchment, P., and Nguyen, L., "Reconstruction and time reversal in thermoacoustic tomography in acoustically homogeneous and inhomogeneous media," Inverse Problems 24(5), 055006 (2008).

[14] Treeby, B. E., Zhang, E. Z., and Cox, B. T., "Photoacoustic tomography in absorbing acoustic media using time reversal," Inverse Problems 26(11), 115003 (2010).

[15] Paltauf, G., Viator, J. A., Prahl, S. A., and Jacques, S. L., "Iterative reconstruction algorithm for optoacoustic imaging," Journal of the Acoustical Society of America 112(4), 1536-1544 (2002).

[16] Zhang, J., Anastasio, M. A., Rivire, P. J. L., and Wang, L. V., "Effects of different imaging models on least-squares image reconstruction accuracy in photoacoustic tomography," IEEE Transactions on Medical Imaging 28(11), 1781-1790 (2009).

[17] Rosenthal, A., Razansky, D., and Ntziachristos, V., "Fast semi-analytical model-based acoustic inversion for quantitative optoacoustic tomography," IEEE Transactions on Medical Imaging 29(6), 1275-1285 (2010).

[18] Deán-Ben, X. L., Buehler, A., Ntziachristos, V., and Razansky, D., "Accurate model-based reconstruction algorithm for three-dimensional optoacoustic tomography," IEEE Transactions on Medical Imaging 31(10), 1922-1928 (2012).

[19] Wang, K., Su, R., Oraevsky, A. A., and Anastasio, M. A., "Investigation of iterative image reconstruction in three-dimensional optoacoustic tomography," Physics in Medicine and Biology 57(17), 5399-5423 (2012).

[20] Prakash, J., Raju, A. S., Shaw, C. B., Pramanik, M., and Yalavarthy, P. K., "Basis pursuit deconvolution for improving model-based reconstructed images in photoacoustic tomography," Biomedical Optics Express 5(5), 1363-1377 (2014).

[21] Dong, Y., Görner, T., and Kunis, S., "An algorithm for total variation regularized photoacoustic imaging," Advances in Computational Mathematics 41(2), 423-438 (2015).

[22] Ding, L., Deán-Ben, X. L., and Razansky, D., "Real-time model-based inversion in cross-sectional optoacoustic tomography," IEEE Transactions on Medical Imaging 35(8), 1883-1891 (2016).

[23] Wang, J., Zhang, C., and Wang, Y., "A photoacoustic imaging reconstruction method based on directional total variation with adaptive directivity," Biomedical Engineering Online 16(1), 64 (2017).

[24] Tick, J., Pulkkinen, A., and Tarvainen, T., "Image reconstruction with uncertainty quantification in photoacoustic tomography," The Journal of the Acoustical Society of America 139(4), 1951-1961 (2016).

[25] Tarantola, A., [Inverse Problem Theory and Methods for Model Parameter Estimation], Society for Industrial and Applied Mathematics (2005).

[26] Kaipio, J. and Somersalo, E., [Statistical and Computational Inverse Problems], Springer Science \& Business Media (2006).

[27] Treeby, B. E. and Cox, B. T., "k-wave: Matlab toolbox for the simulation and reconstruction of photoacoustic wave fields," Journal of Biomedical Optics 15(2), 021314 (2010). 
[28] Arridge, S. R., Betcke, M. M., Cox, B. T., Lucka, F., and Treeby, B. E., "On the adjoint operator in photoacoustic tomography," Inverse Problems 32(11), 115012 (2016).

[29] Rasmussen, C. E. and Williams, C. K. I., [Gaussian Processes for Machine Learning], MIT Press (2006).

[30] Zhang, E., Laufer, J., and Beard, P., "Backward-mode multiwavelength photoacoustic scanner using a planar Fabry-Pérot polymer film ultrasound sensor for high-resolution three-dimensional imaging of biological tissues," Applied Optics 47(4), 561-577 (2008).

[31] Ellwood, R., Ogunlade, O., Zhang, E., Beard, P., and Cox, B., "Photoacoustic tomography using orthogonal Fabry-Pérot sensors," Journal of Biomedical Optics 22(4), 041009-041009 (2017). 